\title{
Lentiviral-mediated overexpression of KCTD12 inhibits the proliferation of human uveal melanoma OCM-1 cells
}

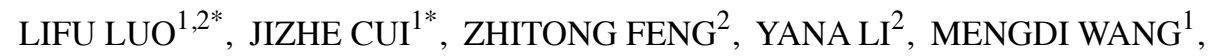 \\ YONG CAI $^{2-4}$, YAZHEN WU ${ }^{1}$ and JINGJI JIN $^{2-4}$ \\ ${ }^{1}$ Department of Ophthalmology, The Second Hospital of Jilin University, Changchun, Jilin 130041; \\ ${ }^{2}$ School of Life Sciences, Jilin University; ${ }^{3}$ National Engineering Laboratory for AIDS Vaccine, Jilin University; \\ ${ }^{4}$ Key Laboratory for Molecular Enzymology and Engineering, The Chinese Ministry of Education, \\ Jilin University, Changchun, Jilin 130012, P.R. China
}

Received June 22, 2016; Accepted August 2, 2016

DOI: $10.3892 / o r .2016 .5325$

\begin{abstract}
Human potassium channel tetramerization domain containing 12 (KCTD12, also known as Pfetin) is a member of the KCTD family which consists of 26 members. It has been reported that KCTD12 regulates agonist potency and kinetics of $\mathrm{GABA}_{\mathrm{B}}$ receptor signaling. Proteomic analysis indicates that KCTD12 may be a potential biomarker for the diagnosis and prognosis of gastrointestinal stromal tumors. However, little has been reported concerning the role of KCTD12 in the other tumor types. In the present study, we designed and subcloned N-terminally Flag-tagged human KCTD12 into the pLVX-Puro vector. We then generated a human uveal melanoma cell line (OCM-1) stably expressing KCTD12. Using this stable cell line, we performed a series of experiments including colony formation, invasion, migration and woundhealing assays, flow cytometry and western blotting. Based on the experimental results, we first demonstrated that KCTD12 effectively suppressed the proliferation of OCM-1 cells and limited the spread of OCM-1 cells. In the flow cytometric analysis, prolongation of the progression of $\mathrm{G} 2 / \mathrm{M}$ to $\mathrm{G} 1$ phase in the KCTD12-overexpressing OCM-1 cells was observed. In addition, inhibition of KCTD12-overexpressing OCM-1 cell xenograft growth in nude mice was observed. Taken together, KCTD12 may serve as a novel therapeutic target for patients with uveal melanoma.
\end{abstract}

Correspondence to: Professor Yazhen Wu, Department of Ophthalmology, The Second Hospital of Jilin University, Changchun, Jilin 130041, P.R. China

E-mail: yazhen_w@163.com

Professor Jingji Jin, School of Life Sciences, Jilin University, Changchun, Jilin 130012, P.R. China

E-mail: jjjin@jlu.edu.cn

${ }^{*}$ Contributed equally

Key words: KCTD12, OCM-1, uveal melanoma, xenograft growth, proliferation

\section{Introduction}

A class of BTB (bric-à-brack, tram-track, broad complex) domain-containing proteins, the KCTD family (potassium channel tetramerization domain protein family which includes 26 members) shares relatively conserved $\mathrm{N}$-terminal domains and variable C-termini (1). However, the KCTD family involved in biological processes including biochemical and structural properties are rather poorly characterized. Potassium channel tetramerization domain containing 12 (KCTD12, also known as Pfetin) belongs to the KCTD family, and was initially identified in human fetal cochlea. KCTD12 is expressed in a variety of fetal organs, particularly with highest expression levels in the cochlea and brain, whereas, it is detected at extremely low levels in adult organs (2). Recent studies have demonstrated that KCTD12 not only stabilizes $\gamma$-aminobutyric acid type $\mathrm{B}\left(\mathrm{GABA}_{\mathrm{B}}\right)$ receptors, which regulate emotionality and neuronal excitability, but also upregulates $\mathrm{GABA}_{\mathrm{B}}$ receptor signaling by stably interacting with $\mathrm{GABA}_{\mathrm{B}}$ receptors (3-5). Using two-dimensional differential gel electrophoresis and mass spectrometry approaches, Suehara et al (6) predicted that KCTD12 may be a prognostic biomarker of gastrointestinal stromal tumors (GISTs). This speculation was confirmed by later investigation. Commercially obtained KCTD12-specific antibody was used for assessing the expression level of KCTD12 in surgical specimens of primary tissues from GIST patients (7). Analyzed data indicate that KCTD12 expression appears more specific for GISTs from neoplastic and non-neoplastic adult tissues other than brain, suggesting the potential predictor of KCTD12 in GISTs (8). Most recently, downregulation of KCTD12 was observed in cancer stem cells (CSCs) in colorectal cancer (CRC). Overexpression of ectopic KCTD12 markedly suppressed CRC cell stemness by inhibiting the extracellular signal-regulated kinase (ERK) pathway, suggesting that KCTD12 may serve as a novel therapeutic target for patients with CRC (9).

So-called uveal melanoma usually refers to melanomas of the choroid, ciliary body and iris of the eye (10). In fact, choroidal and ocular melanomas are the alternative terms of this type of cancer. Uveal melanoma is the most common primary intraocular malignancy in adults. In the US, the 
incident rate of these cancers is $\sim 5 \%, 85 \%$ of which are uveal in origin (11). Patients with uveal melanoma experience painless loss or distortion of vision in the early stage, which even leads to retinal detachment at advanced stage (12). In addition, uveal melanoma is highly metastatic; patients with uveal melanoma are at risk for metastatic disease to the liver, lung and skin $(13,14)$. Therefore, uveal melanoma is associated with high mortality in up to half of uveal-affected patients $(15,16)$. Although the tumorigenesis and the metastasis of uveal melanoma are complex processes (17), genetic mutations such as germline breast cancer 1 (BRCA1)-associated protein 1 (BAP1) (18) and splicing factor 3B subunit 1 (SF3B1) (19), and signaling pathways such as MAPK (20) have been found to be involved in uveal melanoma. However, to date, there have been no studies on the effects of KCTD12 on uveal melanomas. In the present study, we generated an OCM-1 cell line stably expressing Flag-KCTD12 and evaluated the functional properties of KCTD12 in OCM-1 cells.

\section{Materials and methods}

Antibodies. Anti-KCTD12 rabbit polyclonal antibody was purchased from ProteinTech ${ }^{\mathrm{TM}}$ Group (Wuhan, China). AntiE-cadherin, anti- $\beta$-catenin and anti- $\beta$-tubulin polyclonal antibodies were purchased from Ruiying Biological (Suzhou, China). Anti-vimentin, anti-Snail and anti-Slug antibodies were obtained from Wanleibio (Shenyang, China).

Cell culture and maintenance. Human uveal melanoma cell line (OCM-1) purchased from the American Type Culture Collection (ATCC; Manassas, VA, USA) was cultured and maintained in RPMI-1640 medium (Sigma, St. Louis, MO, USA) supplemented with $10 \%$ fetal bovine serum (FBS) (Kangyuan Biology, Tianjin, China) and 1\% penicillin-streptomycin mixture (Thermo Fisher Scientific, Inc., Waltham, MA, USA) at $37^{\circ} \mathrm{C}$ in the presence of $5 \% \mathrm{CO}_{2}$.

Lentiviral vector construction, lentivirus infection and screening. The full length human KCTD12 gene (NM_138444.3) fragment with restriction enzyme cutting site was cloned from 293T cDNA. After the DNA sequence of KCTD12 in the pGEM-T vector was confirmed, the gene fragment was ligated into the pLVX-Puro transfer plasmid (Lenti- $\mathrm{X}^{\mathrm{TM}}$; Clontech Laboratories, Mountain View, CA, USA). The transfer and packaging plasmids (pRRE, pRSV-Rev and pCMV-VSVG) were mixed at the quality ratio of 800:400:140:200. 293T cells cultured in $6-\mathrm{cm}$ plates were then transfected with $5 \mu \mathrm{g}$ of DNA plasmids using Lipofectamine 2000 reagent (Invitrogen, Carlsbad, CA, USA). The medium was replaced after $24 \mathrm{~h}$, and then the cell supernatants were collected by centrifugation at $10,000 \mathrm{xg}$ for $10 \mathrm{~min}$ at room temperature and stored at $-80^{\circ} \mathrm{C}$. OCM-1 cells were infected with a double volume of recombinant viruses and the cells were selected with $1 \mu \mathrm{g} / \mathrm{ml}$ puromycin for 2 weeks according to the manufacturer's protocol. The antibiotic-resistant colonies were then expanded for further analysis. Stably expressing Flag-KCTD12 cell line was confirmed by western blotting and immunofluorescence staining.

Immunofluorescence staining. Stably expressing FlagKCTD12 cells in 24-well plates containing a coverslip (8D1007,
Nest) on each well were grown to $80 \%$ confluence. Then, the cells were washed with phosphate-buffered saline (PBS) buffer, fixed with $4 \%$ paraformaldehyde (PFA) for $15 \mathrm{~min}$ at room temperature, and permeabilized in $0.5 \%$ Triton X-100 in PBS buffer for $5 \mathrm{~min}$. Cells were then incubated with the KCTD12 primary antibody (1:500) at room temperature, and stained with the FITC-conjugated secondary antibody (rabbit/green 1:300; sc-2012). Cell nuclei were stained using DAPI containing Vectashield (Vector Laboratories, Inc., Burlingame, CA, USA). Fluorescence images were observed with an Olympus BX40F microscope (Olympus Corporation, Tokyo, Japan).

MTS assays. Cells $\left(\sim 3 \times 10^{3}\right)$ were plated into 96 -well plates. After $48 \mathrm{~h}$, the MTS [3-(4,5-dimethylthiazol-2-yl)-5-(3carboxymethoxyphenyl)-2-(4-sulfophenyl)-2H-tetrazolium] (CellTiter 96; Promega, Madison, WI, USA) solution was added into the wells and incubated at $37^{\circ} \mathrm{C}$ for $1 \mathrm{~h}$. The absorbance was measured at a wavelength of $490 \mathrm{~nm}$ with a microplate reader (Bio-Rad Laboratories, Hercules, CA, USA).

Colony formation assays. Cells $\left(\sim 10^{3}\right)$ were seeded into 24-well plates. After 10 days of culture, formed colonies were stained with $0.1 \%$ crystal violet. Colonies containing $>20$ cells were scored as positive. Colonies were photographed by Gel Imaging System (Liuyi Instrument Plant, Beijing, China).

Soft agar assays. Briefly, $5 \times 10^{3}$ cells were resuspended in medium containing $10 \%$ FBS with $0.3 \%$ agarose, and layered on top of $0.6 \%$ agar in medium supplemented with $20 \%$ FBS on $35-\mathrm{mm}$ plates. After 14 days of culture, the cells were stained with $0.05 \%$ crystal violet for $1 \mathrm{~h}$. Colonies were photographed and the number of foci $>100 \mu \mathrm{m}$ was counted.

Wound healing assay. Cells were cultured in 6-well plates and wounds were introduced by scraping the confluent cell cultures with the tip of a $10-\mu 1$ pipette. Floating cells were removed and medium without serum was added. The wound healing process was monitored under a microscope.

Migration and invasion assays. For the migration and invasion assays, 24-well Transwell chambers (Corning Inc., Corning, NY, USA) were used. Filters ( $8-\mu \mathrm{m}$ pore size) were used for estimating cell migration, and filters coated with Matrigel (BD Biosciences, Franklin Lakes, NJ, USA) were used for estimating cell invasion. Cells in $0.1 \mathrm{ml}$ of serum-free RPMI-1640 medium were placed into the upper chamber $\left(0.5 \times 10^{5}\right.$ cells/filter $)$. Complete medium was placed in the lower chamber as a chemoattractant. Migration and invasion were scored at $24 \mathrm{~h}$ after cells were seeded. Cells were fixed in methanol for $5 \mathrm{~min}$ at room temperature, stained with crystal violet for $5 \mathrm{~min}$, and counted under microscopy. The mean numbers of cells/microscopic field over 5 fields/filter were calculated.

In vivo tumorigenicity experiments. Male BALB/c nude mice (6-week old, 15-17 g) were randomly divided into 2 groups ( $\mathrm{n}=3$ /group) for the OCM-1 cell xenograft growth experiment. For tumor cell implantation, the OCM-1 cells stably expressing Flag-KCTD12 or a vector control $\left(1 \times 10^{6}\right)$ suspended in $100 \mu 1$ PBS were injected into the armpits of the mice. The weight 
A

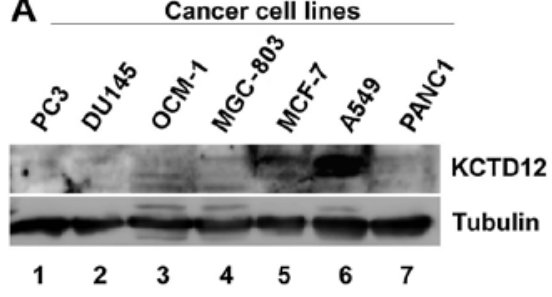

C

Subcloned KCTD12 into pLVX-Puro<smiles>C1CC(C2CC2)C1</smiles>

Packaging virus in 293T cells

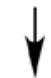

OCM-1 cells were infected by virus

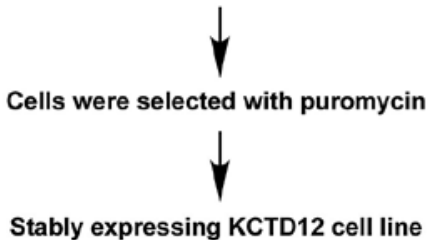

B

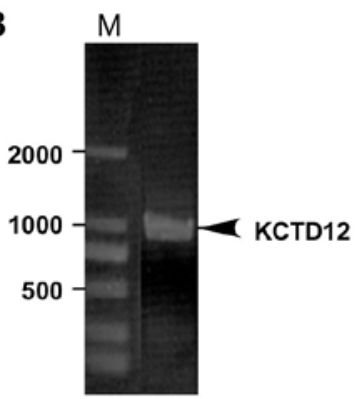

E

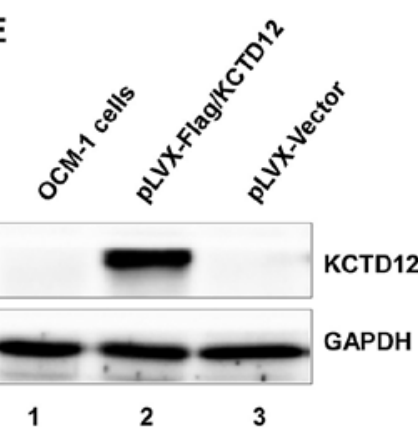

D 293T cells

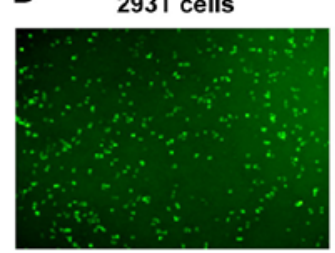

pLVX-GFP
F OCM-1 stable cell line

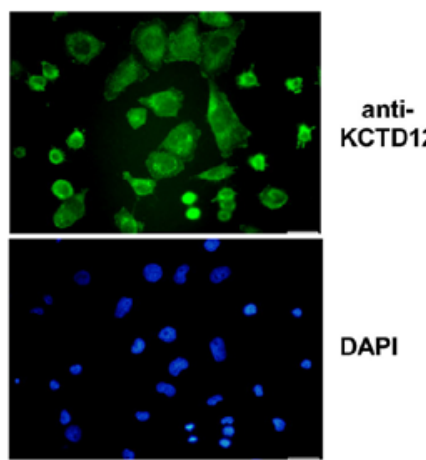

Figure 1. Generation of the stably expressing Flag-KCTD12 OCM-1 cell line. (A) Expression of KCTD12 in different cancer cell lines. Western blot analysis was performed to detect endogenous KCTD12 in the indicated cancer cell lines. (B) PCR amplification of the KCTD12 gene from cDNA. The arrow indicates the specific KCTD12 DNA fragment (977 bp). (C) Procedural steps to establish the stably expressing KCTD12 OCM-1 cell line. (D) Confirmation of the packaging virus. $293 \mathrm{~T}$ cells were infected by a lentivirus which contains a fluorescent protein. The generated stably expressing KCTD12 OCM-1 cell line was analyzed by (E) western blotting and (F, upper panel) immunofluorescence staining. (F, lower panel) DAPI staining showing total nuclei.

of mouse and the volume $(\mathrm{V})$ of tumors were examined ( $\mathrm{V}=$ tumor length $\mathrm{x}$ width ${ }^{2} \mathrm{x}$ 0.5236). All experiments were performed in accordance with the Institutional Animal Care and Use Committee of Jilin University.

Cell cycle analysis. OCM-1 cells stably expressing Flag-KCTD12 or a vector control were grown to $80-90 \%$ confluency. In the cell cycle arrest experiment, as previously described, cells were synchronized by treatment with $1 \mathrm{mM}$ hydroxyurea (HU) for $24 \mathrm{~h}$, and were then harvested by trypsinization $0,2,4,6,8,10$ and $18 \mathrm{~h}$ after removal of HU (21). Data collection was performed using EPICS XL ${ }^{\mathrm{TM}}$ flow cytometer (Beckman Coulter, Brea, CA, USA). Acquired data were analyzed using FlowJo software. The experiment was repeated 3 times under the same conditions.

Analysis of apoptosis. Apoptosis in OCM-1 cells was determined by an Annexin V-fluorescein isothiocyanate (FITC) staining kit according to the manufacturer's protocol (KeyGen Biotec, Nanjing, China). Propidium iodide (PI) was used to differentiate apoptotic cells with membrane integrity (Annexin $\mathrm{V}^{+} / \mathrm{PI}^{-}$) from necrotic cells that had lost their membrane integrity (Annexin $\mathrm{V}^{+} / \mathrm{PI}^{+}$). The percentage of apoptotic cells was calculated using an Accuri Cytometer and the data were analyzed by CFlow software.

Statistical analysis. All the data were analyzed via the Student's t-test (Microsoft Excel). The statistical difference of $\mathrm{p}<0.05$ was considered to indicate a statistically significant result.

\section{Results}

Stably expressing Flag-KCTD12 is established in OCM-1 cells. To determine the expression levels of KCTD12 in cancer cells, we first analyzed the endogenous KCTD12 protein levels in different cancer cell lines. As shown in Fig. 1A, except for lung carcinoma type II epithelium-like A549 cells, KCTD12 protein levels in other cancer cell lines including prostate cancer PC3 and DU145 cells, OCM-1, gastric cancer MGC-803 cells, human breast adenocarcinoma MCF-7 cells, and pancreatic cancer cell PANC1 cells were extremely low. In order to further investigate the roles of KCTD12 in cancer cells, OCM-1 cells were chosen as a test subject. Amplified PCR products of KCTD12 (Fig. 1B) were subcloned into the pLVX-Puro vector following the procedure (Fig. 1C and D) to establish the stably expressing Flag-KCTD12 OCM-1 cell line. The established stable cell line was successfully confirmed by western blotting (Fig. 1E) and immunofluorescence staining (Fig. 1F).

Overexpression of KCTD12 suppresses the proliferation of OCM-1 cells. Using the established stable cell line, we first performed the MTS assays. As shown in Fig. 2C, attenuated viability was observed in the KCTD12-overexpressing cells $(\mathrm{p}<0.05)$. Furthermore, to explore the functions of KCTD12 in the proliferation and tumorigenesis of OCM-1 cells, colony formation and soft-agar assays were performed in vitro. In both assays, significantly decreased colony formation (Fig. 2A, upper images) and much smaller colony size (Fig. 2B) were observed in the KCTD12-overexpressing 

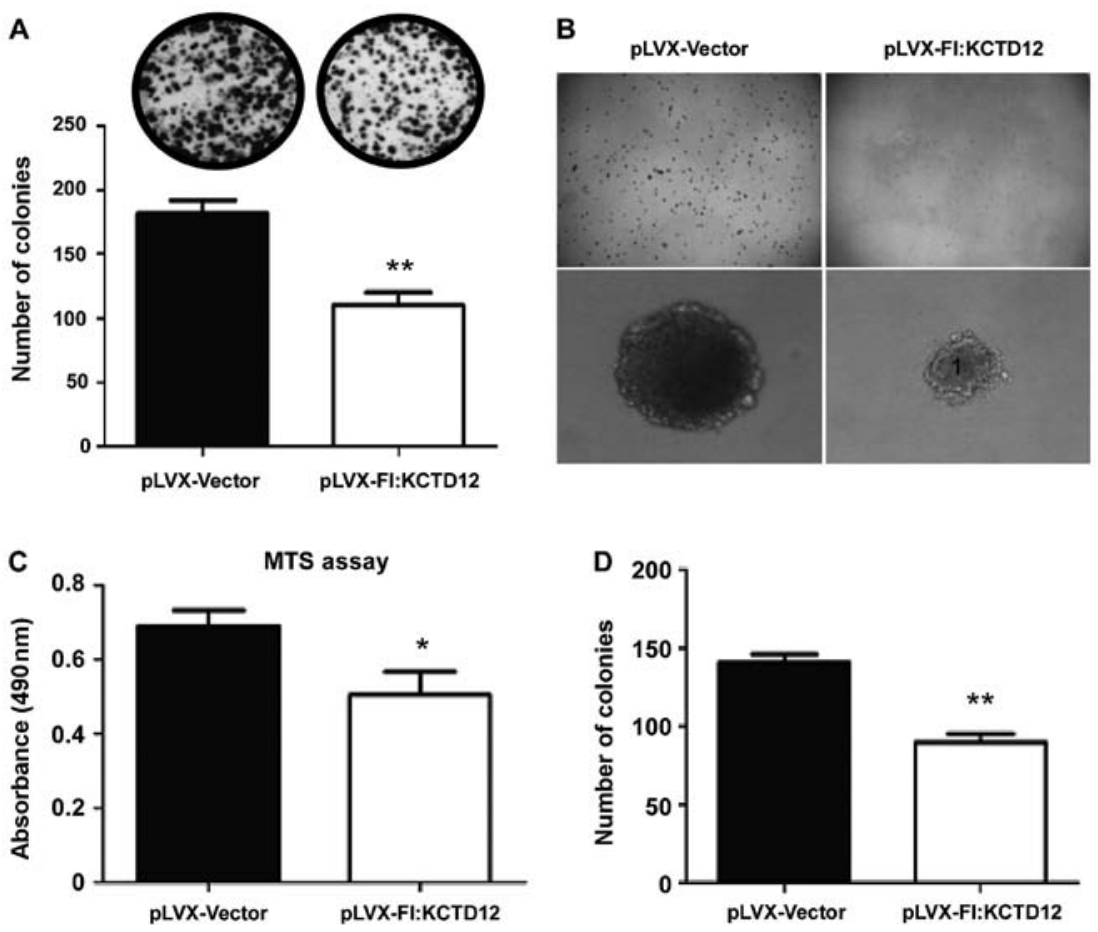

Figure 2. Overexpression of KCTD12 suppresses OCM-1 cell proliferation. (A) Colony formation assay ( $\mathrm{n}=3$ ). Colony formation assay was performed using the indicated stable cell lines. Counted colonies are shown as a bar graph. (B) Soft-agar formation assay $(n=3)$. Three independent soft agar colony formation assays were performed. Images of the colonies produced by OCM-1 stably expressing KCTD12 and pLVX-vector cells were captured under a microscope before staining (upper). Lower images show the enlarged colonies from the different cell lines. (C) MTS assay $(n=3)$. The cell proliferation was measured by MTS in the indicated stable cell lines. (D) Numbers of colonies. Images of stained colonies were captured and the number of foci $>100 \mu \mathrm{m}$ was counted. Counted numbers of focis are shown as a bar graph; ${ }^{*} \mathrm{p}<0.05,{ }^{* *} \mathrm{p}<0.01$ (Student's t-test).
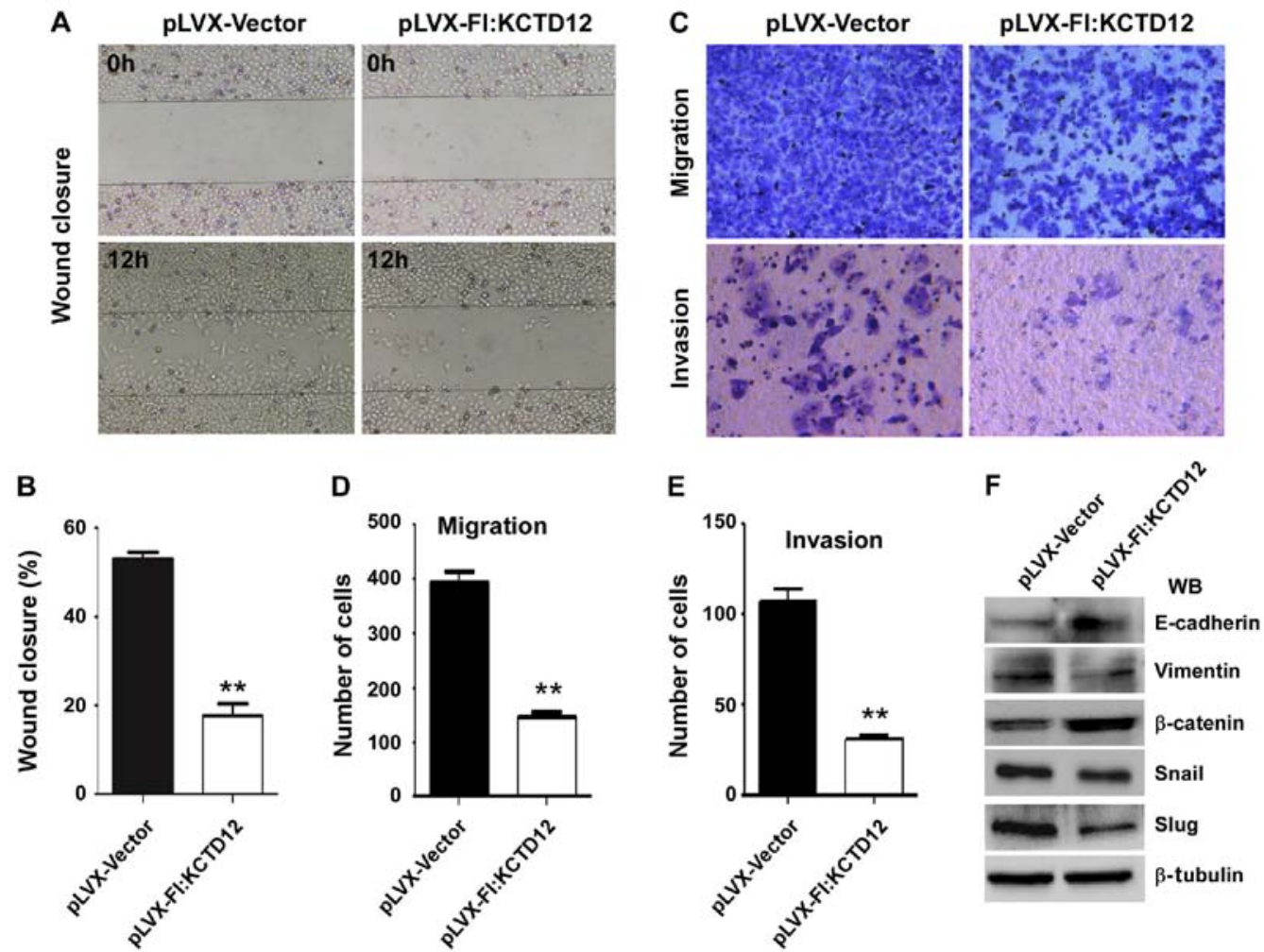

Figure 3. Overexpression of KCTD12 suppresses OCM-1 cell migration and invasion. (A) Wound healing assay. Scratch wound healing assay was performed in vector and KCTD12 stably expressing OCM-1 cells. Images were captured at 0 and $12 \mathrm{~h}$ after initiation of the scratch wound. (B) Percentage of wound closure is shown as a bar graph. (C) Migration and invasion assays. The 24-well Transwell chambers were used for migration and invasion assays. Twenty-four hours after the cells were added, cells were stained with crystal violet and counted under a microscope. (D and E) Migration/invasion of cells from $>5$ fields/filter/microscopic field were calculated. Bar graph shows the mean $\pm \mathrm{SD}(\mathrm{n}=3) ;{ }^{* *} \mathrm{p}<0.01$ (Student's $\mathrm{t}$-test). (F) Western blotting. Levels of EMT-related proteins were detected by western blotting with specific antibodies. 
A
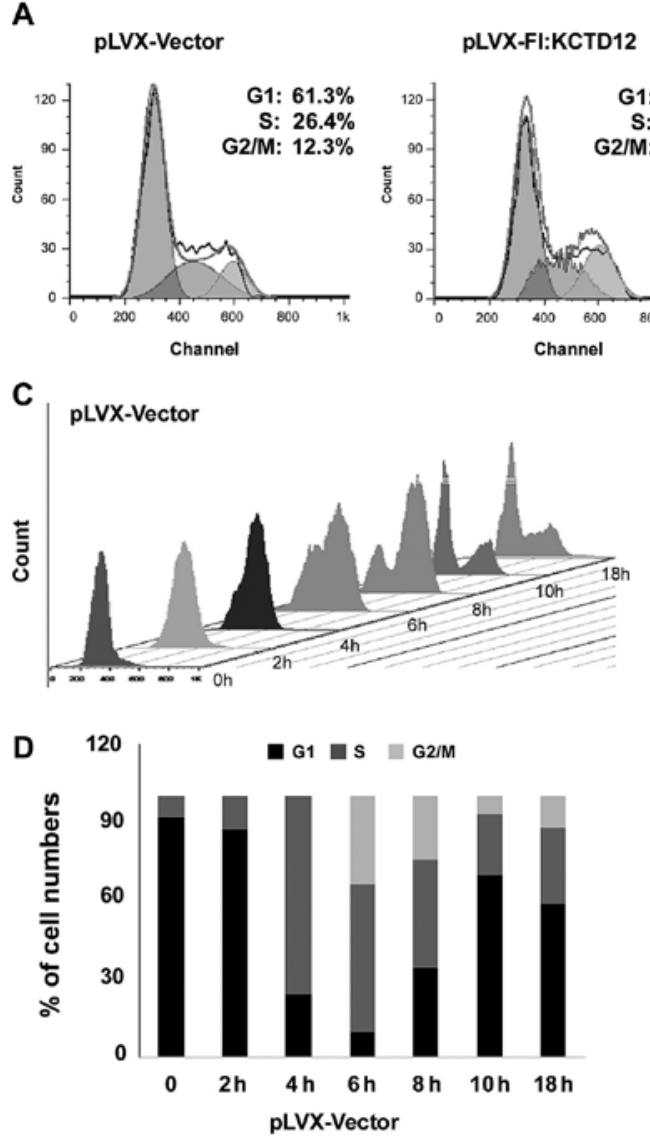
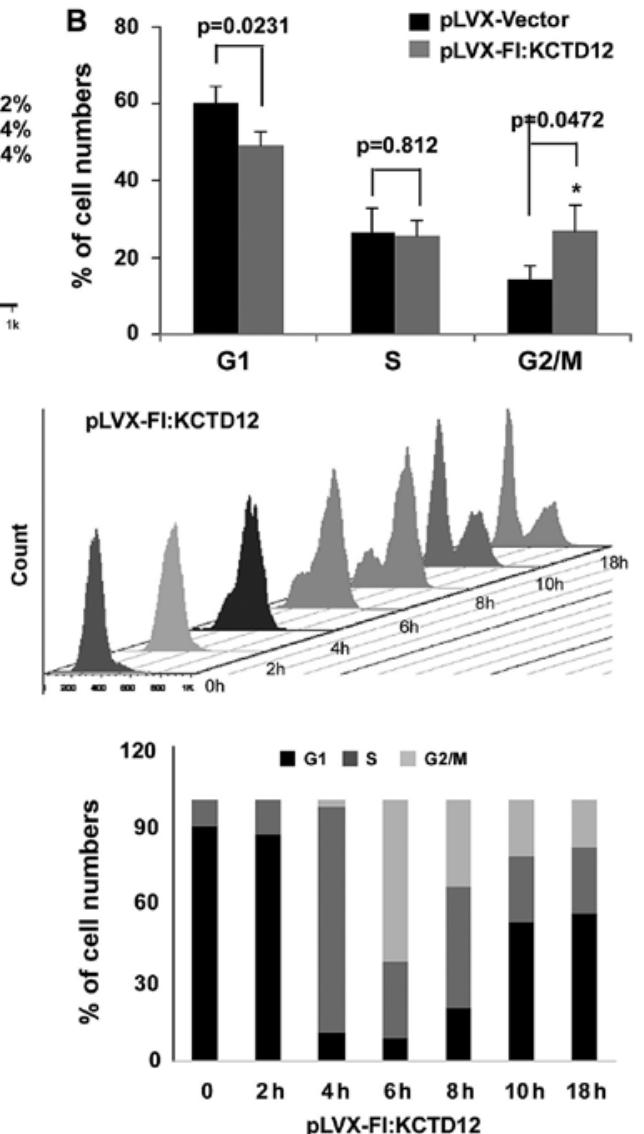

Figure 4. Delayed progression of G2/M occurs in the KCTD12-expressing stable cell line. (A) Flow cytometric analysis of the cell cycle distribution of the KCTD12 stably expressing OCM-1 cell line. Fluorescence-activated cell sorting (FACS) analysis of propidium iodide-stained cells was performed (B) Analysis of the subpopulation of cells in cell cycle phases G1, S and G2/M. Data shown are the means \pm SD of a representative experiment performed in triplicate ( $\mathrm{n}=3) ;{ }^{*} \mathrm{p}<0.05$ in comparison with the pLVX-Vector cells (Student's t-test). (C) Delayed progression of G2/M phase in the KCTD12 stably expressing cells. pLVX-Vector (left) or KCTD12 stably expressing cells (right) were synchronized by treatment with $1 \mathrm{mM}$ HU for a 24-h incubation. Cells were then harvested $0,2,4,6,8,10$, and $18 \mathrm{~h}$ after removal of HU. Acquired data were analyzed using FlowJo software. (D) Quantified cell cycle distribution in pLVX-Vector (left) or KCTD12 stably expressing cells (right).

OCM-1 cells. Significant difference in colony numbers between the KCTD12-overexpressing and vector controls OCM-1 cells was found ( $\mathrm{p}<0.01$ in both assays) (Fig. 2A, lower, and D).

Overexpression of KCTD12 inhibits OCM-1 cell motility. To further observe the effects of KCTD12 on OCM-1 cell motility, we performed wound-healing assays. The images after $12 \mathrm{~h}$ of the initial scratch wound are shown in Fig. 3A. The progression of wound closure in the KCTD12 stably expressing OCM-1 cells was much slower than that in the control cells. Compared to the control cells, the percentage of wound closure was significantly decreased $(\mathrm{p}<0.01)$ (Fig. 3B). Then, Transwell migration (Fig. 3C, upper) and invasion assays (Fig. 3C, lower) were carried out to examine the roles of KCTD12 in metastasis. Quantified number of cells which penetrated the membrane was largely decreased compared with the control cells ( $\mathrm{p}<0.01)$ (Fig. 3D). Similarly, overexpression of KCTD12 markedly reduced the invasive ability of the OCM-1 cells $(\mathrm{p}<0.01)$ (Fig. 3E). Western blot analysis revealed that the expression levels of various epithelial-mesenchymal transition (EMT)-related molecules were altered. As shown in Fig. 3F, elevated E-cadherin and $\beta$-catenin protein levels and decreased mesenchymal (MSC) markers vimentin, Snail and Slug were noted in the KCTD12-overexpressing OCM-1 cells.
Overexpression of KCTD12 prolongs the progression of the G2/M phase and induces apoptosis in the OCM-1 cells. Given that overexpression of KCTD12 significantly inhibits OCM-1 cell proliferation, we speculated that KCTD12 is involved in cell cycle progression. Thus, FACS analysis was performed on KCTD12-overexpressing or control OCM-1 cells. Compared to the control cells, stably expressing KCTD12 cells had delayed G2/M phase progression (Fig. 4A). The percentages of the subpopulation of cells in cell cycle phases G1, S and G2/M are shown in Fig. 4B. The percentage of KCTD12-expressing cells in cell cycle phase G2/M was statistically increased compared to the percentage in the pLVX-Vector cells $(\mathrm{p}<0.05)$. To further confirm this observation, both cells were treated with $1 \mathrm{mM}$ HU to block cells at the G1/S phase, so that, no new G2/M cells could be generated. Cell flow cytometry and FACS analysis in both cell lines were performed at 0 , 2, 4, 6, 8, 10 and $18 \mathrm{~h}$ after removal of HU (Fig. 4C). The gradual increase in the $\mathrm{G} 2 / \mathrm{M}$ phase population was observed in the KCTD12-overexpressing cells (Fig. 4D). To further confirm whether overexpression of KCTD12 can affect cell apoptosis, flow cytometry of Annexin V binding/PI uptake was assessed in the KCTD12-overexpressing cell line and vector only control (Fig. 5A). Apoptosis is depicted in Fig. 5B. Overexpression of KCTD12 in OCM-1 cells significantly 
A

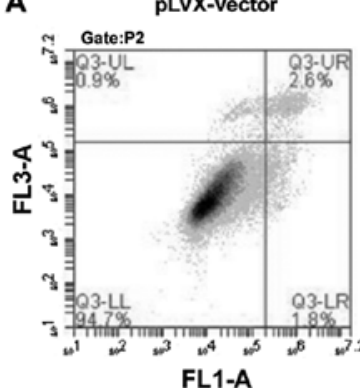

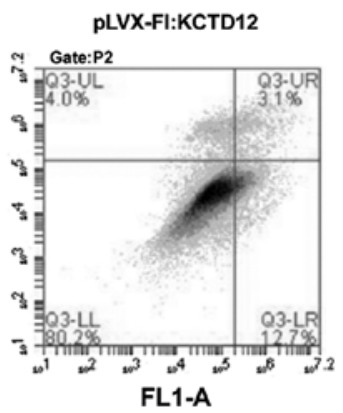
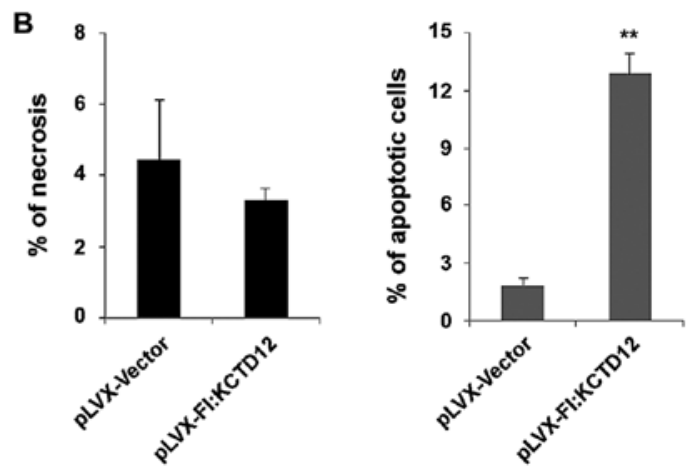

Figure 5. Significant apoptosis is induced in the KCTD12-overexpressing OCM-1 cells. (A) Annexin V/PI-FACS analysis. pLVX-Vector and KCTD12 stably expressing cells were stained with Annexin V-FITC and propidium iodide and analyzed by FACS. Numbers in the upper left and right, lower left and right quadrants represent the percentage of damaged, necrotic, live and apoptotic cells, respectively. (B) Quantified percentages of necrotic and apoptotic cells in A; *** $\mathrm{p}<0.01$ (Student's t-test), statistical significant difference.
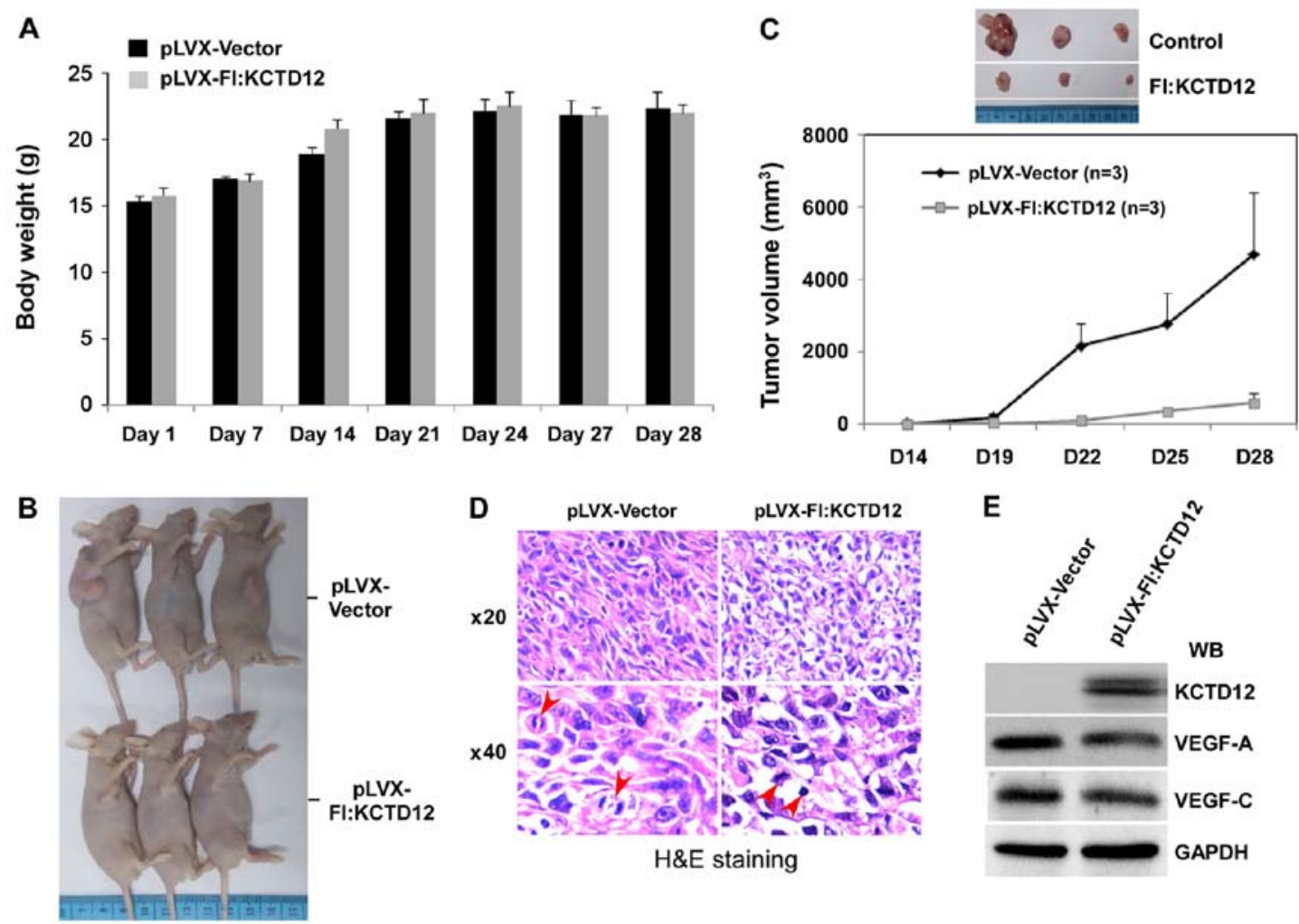

Figure 6. Effect of the suppression of KCTD12 on OCM-1 cell xenograft growth. (A) Changes in body weight. Weight tracking was performed at indicated time point. (B) The images of mice harboring tumors. A xenograft model consisting of 6 -week-old nude mice ( $\mathrm{n}=3 / \mathrm{group}$ ) injected into the armpits with stably expressing KCTD12 OCM-1 cells. (C) The images of tumors from the mice (upper) and graph of the tumor volumes. Tumor volumes were determined at the indicated days (lower). (D) H\&E staining of tumor tissue sections. Original magnification, x200. (E) Western blot analysis. Indicated specific proteins were detected by western blotting with specific antibodies.

increased apoptosis compared to that in the vector only control $(p<0.01)$ (Fig. 5B, right). However, there was no significant statistical difference in necrotic cells between the vector and KCTD12-overexpressing group ( $\mathrm{p}>0.05$ ) (Fig. 5B, left).

Overexpression of KCTD12 restricts OCM-1 cell xenograft growth. The above-described series of in vitro experiments confirmed the effective inhibition of the cell growth of KCTD12 in OCM-1 cells. To further verify the functions of KCTD12 on the proliferation and tumorigenesis of OCM-1 cells, a xenograft model consisting of BALB/c nude mice was designed. Stably expressing KCTD12 OCM-1 cells were injected into the armpits of 6-week mice, and the cells were allowed to grow 28 days. During this time, the body weight of the mice between the vector and KCTD12-overexpressing groups had no obvious difference (Fig. 6A). However, overexpression of KCTD12 significantly blocked OCM-1 cell xenograft growth (Fig. 6B). As shown in Fig. 6C, the OCM-1 cell xenograft growth was slower in the KCTD12overexpressing group than that noted in the vector control 
group (lower). In addition, differences in tumor size between the two groups are visualized in Fig. 6C (upper images). Cell and nuclear morphology of the tumor tissues was visualized by hematoxylin and eosin $(\mathrm{H} \& \mathrm{E})$ staining. Microscopic images of H\&E staining are shown in Fig. 6D. Morphological integrity of the cells and a plurality of cells in the mitotic phase can be seen in the vector control group (left panel). In contrast, irregularly shaped cells and nuclear condensation were found in the stably expressing KCTD12 OCM-1 cell group (right panel). In addition, decreased vascular endothelial growth factor VEGF-A and VEGF-C were detected by western blot analysis using prepared whole cell lysate from the tumor tissues (Fig. 6E), suggesting the involvement of KCTD12 in regulating $V E G F$.

\section{Discussion}

In the present study, we first clarified that lentiviral-mediated overexpression of KCTD12 inhibits tumor proliferation in human uveal melanoma OCM-1 cells using both in vitro and in vivo approaches. A series of in vitro assays including MTS, colony formation, migration and invasion assays presented evidence that stably expressing Flag-tagged KCTD12 significantly suppressed the proliferation and tumorigenesis of OCM-1 cells. In addition, overexpression of KCTD12 inhibited tumor growth in a xenograft model.

The KCTD proteins with sequence similarity between its $\mathrm{N}$-terminal region and the tetramerization domain in some voltage-gated potassium channels are involved in various cellular biological processes (22). For example, KCDT proteins as adaptor molecules interact with the Cul3 ubiquitin ligase and its substrate $(23,24)$. In addition, increasing evidence suggests that the KCTD family is implicated in proliferation, differentiation, apoptosis and metabolism of certain cancer cells $(25,26)$. Based on alignment of the amino acids in the potassium tetramerization domains, KCTD proteins can be divided into 7 groups of A to G (22). KCTD12 with KCTD8 and KCTD16 together make up the D-group that directly binds to the $\mathrm{GABA}_{\mathrm{B}}$ receptor and an associated $\mathrm{G}$ protein $(3,4)$. KCTD12 as a potential prognostic biomarker of GISTs was screened by 2-D gel combined mass spectrometry approaches. Later studies confirmed that KCTD12 produced desensitization of the channel to $\mathrm{GABA}_{\mathrm{B}}$ receptor signaling by inhibiting the interaction of $G \beta \gamma$ and Kir3 channels, thereby suppressing proliferation in GISTs (8). Although the precise mechanism is unclear, our experimental results verified that overexpression of KCTD12 markedly suppressed the proliferation of OCM-1 cells. Stable expression of KCTD12 in OCM-1 cells decreased the cell viability in MTS assays, and reduced colony numbers in colony formation and soft agar assays, suggesting the role of KCTD12 in the proliferation of OCM-1 cells (Fig. 2). Furthermore, overexpression of KCTD12 in OCM-1 cells not only prolonged the $\mathrm{G} 2 / \mathrm{M}$ phase population (Fig. 4), but also increased apoptosis (Fig. 5), suggesting that the ability of KCTD12 to inhibit OCM-1 cell proliferation may be partly due to the delay in the cell cycle which causes apoptosis.

A recent study demonstrated that KCTD12 is involved in the regulation of colorectal cancer cell (CRC) stemness. Silencing of KCTD12 markedly enhanced CRC cell stemness, while overexpression of KCTD12 repressed CRC cell stemness and its markers such as CD44, CD133 and CD29 (9). In the present study, overexpression of KCTD12 markedly suppressed OCM-1 cell xenograft growth in nude mice (Fig. 6). Based on $\mathrm{H} \& \mathrm{E}$ staining of grown tumor tissues, increased numbers of irregularly shaped and cells with nuclear condensation were found in the KCTD12-overexpressing OCM-1 cell group. In addition, decreased VEGF-A and VEGF-C were detected by western blot analysis. Taken together, KCTD12 is implicated in the tumorigenesis of OCM-1 cells.

It has been found that uveal melanoma is highly metastatic. Thus, patients with uveal melanoma are at risk of developing metastatic disease to other organs. Epithelial-mesenchymal transition (EMT) is a critical process driving the early phase of cancer metastasis. The process of EMT can be regulated by many transcription factors. For example, loss of E-cadherin and $\beta$-catenin can trigger the EMT process (27). In contrast, activation of Snail and Slug often occur in EMT (28). In the present study, overexpression of KCTD12 significantly restricted the migration and invasion of OCM-1 cells in in vitro assays. In addition, an increase in E-cadherin and $\beta$-catenin, and a reduction in Snail and Slug were confirmed by western blot analysis (Fig. 3), suggesting the roles of KCTD12 in inhibiting the metastasis of uveal melanoma by regulating the above genes.

In conclusion, although further research is needed to clarify the precise mechanisms of uveal melanoma, our data demonstrated that lentiviral-mediated overexpression of KCTD12 suppressed the proliferation and metastasis of uveal melanoma OCM-1 cells in vitro and in vivo, suggesting that KCTD12 plays an important role in the tumorigenesis of uveal melanoma, and may serve as a novel therapeutic target for patients with uveal melanoma.

\section{Acknowledgements}

The present study was supported by the National Natural Science Foundation of China (nos. 31371311 and 31571316), by the National Laboratory of Biomacromolecules (O5SY02110A and 2012kf04), and by the Project of Jilin Province Science and Technology Development Program (20130413002GH and 20140414057GH).

\section{References}

1. Ji AX, Chu A, Nielsen TK, Benlekbir S, Rubinstein JL and Privé GG: Structural insights into KCTD protein assembly and cullin3 recognition. J Mol Biol 428: 92-107, 2016.

2. Resendes BL, Kuo SF, Robertson NG, Giersch AB, Honrubia D, Ohara O, Adams JC and Morton CC: Isolation from cochlea of a novel human intronless gene with predominant fetal expression. J Assoc Res Otolaryngol 5: 185-202, 2004.

3. Schwenk J, Metz M, Zolles G, Turecek R, Fritzius T, Bildl W, Tarusawa E, Kulik A, Unger A, Ivankova K, et al: Native GABA(B) receptors are heteromultimers with a family of auxiliary subunits. Nature 465: 231-235, 2010.

4. Turecek R, Schwenk J, Fritzius T, Ivankova K, Zolles G, Adelfinger L, Jacquier V, Besseyrias V, Gassmann M, Schulte U, et al: Auxiliary $\mathrm{GABA}_{\mathrm{B}}$ receptor subunits uncouple $\mathrm{G}$ protein $\beta \gamma$ subunits from effector channels to induce desensitization. Neuron 82: 1032-1044, 2014.

5. Cathomas F, Stegen M, Sigrist H, Schmid L, Seifritz E, Gassmann M, Bettler B and Pryce CR: Altered emotionality and neuronal excitability in mice lacking KCTD12, an auxiliary subunit of $\mathrm{GABA}_{\mathrm{B}}$ receptors associated with mood disorders. Transl Psychiatry 5: e510, 2015. 
6.Suehara Y, Kondo T, Seki K, Shibata T, Fujii K, Gotoh M, Hasegawa T, Shimada Y, Sasako M, Shimoda T, et al: Pfetin as a prognostic biomarker of gastrointestinal stromal tumors revealed by proteomics. Clin Cancer Res 14: 1707-1717, 2008.

7. Kubota D, Mukaihara K, Yoshida A, Suehara Y, Saito T, Okubo T, Gotoh M, Orita H, Tsuda H, Kaneko K, et al: The prognostic value of pfetin: A validation study in gastrointestinal stromal tumors using a commercially available antibody. Jpn J Clin Oncol 43: 669-675, 2013.

8. Hasegawa T, Asanuma H, Ogino J, Hirohashi Y, Shinomura Y, Iwaki $\mathrm{H}$, Kikuchi $\mathrm{H}$ and Kondo $\mathrm{T}$ : Use of potassium channel tetramerization domain-containing 12 as a biomarker for diagnosis and prognosis of gastrointestinal stromal tumor. Hum Pathol 44: 1271-1277, 2013.

9. Li L, Duan T, Wang X, Zhang RH, Zhang M, Wang S, Wang F, $\mathrm{Wu}$ Y, Huang $\mathrm{H}$ and Kang T: KCTD12 regulates colorectal cancer cell stemness through the ERK pathway. Sci Rep 6: 20460, 2016

10. Chattopadhyay C, Kim DW, Gombos DS, Oba J, Qin Y, Williams MD, Esmaeli B, Grimm EA, Wargo JA, Woodman SE, et al: Uveal melanoma: From diagnosis to treatment and the science in between. Cancer 122: 2299-2312, 2016.

11. Chang AE, Karnell LH and Menck HR; The American College of Surgeons Commission on Cancer and the American Cancer Society: The National Cancer Data Base report on cutaneous and noncutaneous melanoma: A summary of 84,836 cases from the past decade. Cancer 83: 1664-1678, 1998

12. Eskelin $\mathrm{S}$ and Kivelä T: Mode of presentation and time to treatment of uveal melanoma in Finland. Br J Ophthalmol 86: 333-338, 2002.

13. Shields CL and Shields JA: Ocular melanoma: Relatively rare but requiring respect. Clin Dermatol 27: 122-133, 2009.

14. Singh AD, Turell ME and Topham AK: Uveal melanoma: Trends in incidence, treatment, and survival. Ophthalmology 118: 1881-1885, 2011.

15. Singh AD and Topham A: Survival rates with uveal melanoma in the United States: 1973-1997. Ophthalmology 110: 956-961, 2003.

16. Gill HS and Char DH: Uveal melanoma prognostication: From lesion size and cell type to molecular class. Can J Ophthalmol 47: 246-253, 2012.

17. Patel SP: Latest developments in the biology and management of uveal melanoma. Curr Oncol Rep 15: 509-516, 2013.
18. Harbour JW, Roberson ED, Anbunathan H, Onken MD, Worley LA and Bowcock AM: Recurrent mutations at codon 625 of the splicing factor SF3B1 in uveal melanoma. Nat Genet 45: 133-135, 2013.

19. Field MG and Harbour JW: Recent developments in prognostic and predictive testing in uveal melanoma. Curr Opin Ophthalmol 25: 234-239, 2014.

20. Yu FX, Zhang K and Guan KL: YAP as oncotarget in uveal melanoma. Oncoscience 1: 480-481, 2014.

21. Cao L, Ding J, Dong L, Zhao J, Su J, Wang L, Sui Y, Zhao T, Wang F, Jin J, et al: Negative regulation of $\mathrm{p} 21^{\text {Waf } / / \text { Cipl }}$ by human INO80 chromatin remodeling complex is implicated in cell cycle phase G2/M arrest and abnormal chromosome stability. PLoS One 10: e0137411, 2015.

22. Liu Z, Xiang Y and Sun G: The KCTD family of proteins: Structure, function, disease relevance. Cell Biosci 3: 45, 2013.

23. Canettieri G, Di Marcotullio L, Greco A, Coni S, Antonucci L, Infante P, Pietrosanti L, De Smaele E, Ferretti E, Miele E, et al: Histone deacetylase and Cullin3-REN ${ }^{\mathrm{KCTD} 11}$ ubiquitin ligase interplay regulates Hedgehog signalling through Gli acetylation. Nat Cell Biol 12: 132-142, 2010.

24. Staropoli JF, Karaa A, Lim ET, Kirby A, Elbalalesy N, Romansky SG, Leydiker KB, Coppel SH, Barone R, Xin W, et al: A homozygous mutation in KCTD7 links neuronal ceroid lipofuscinosis to the ubiquitin-proteasome system. Am J Hum Genet 91: 202-208, 2012.

25. Mancarelli MM, Zazzeroni F, Ciccocioppo L, Capece D, Po A, Murgo S, Di Camillo R, Rinaldi C, Ferretti E, Gulino A, et al: The tumor suppressor gene KCTD $11^{R E N}$ is regulated by $\mathrm{Sp} 1$ and methylation and its expression is reduced in tumors. Mol Cancer 9: 172, 2010.

26. Faryna M, Konermann C, Aulmann S, Bermejo JL, Brugger M, Diederichs S, Rom J, Weichenhan D, Claus R, Rehli M, et al: Genome-wide methylation screen in low-grade breast cancer identifies novel epigenetically altered genes as potential biomarkers for tumor diagnosis. FASEB J 26: 4937-4950, 2012.

27. Xu Q, Deng F, Qin Y, Zhao Z, Wu Z, Xing Z, Ji A and Wang QJ: Long non-coding RNA regulation of epithelial-mesenchymal transition in cancer metastasis. Cell Death Dis 7: e2254, 2016.

28. Yang J and Weinberg RA: Epithelial-mesenchymal transition: At the crossroads of development and tumor metastasis. Dev Cell 14: 818-829, 2008. 